\title{
Mode Selection Mechanism to Enable Effective Device-to- Device Communication System over Different Environments
}

\author{
https://doi.org/10.3991/ijim.v13i04.10518 \\ Feras Zen Alden $\left.{ }^{(}\right)$, Suhaidi Hassan, Adib Habbal \\ Universiti Utara UUM, Kedah, Malaysia \\ yasoda1516@gmail.com
}

\begin{abstract}
Device-to-Device (D2D) communication is an important component of the $5 \mathrm{G}$ mobile networks. D2D communication enables users to communicate either directly without network assistance or with minimum signalling information through a base station (BS). Hence, D2D communication can enhance system capacity, increase spectral efficiency, improve throughput and reduce latency. One of the main challenges in D2D communications that when a potential D2D pair can switch between direct and conventional cellular communications, there lies a challenge in identifying D2D mode selection between communicating devices (i.e. a D2D pair). This paper aims to evaluate the mode selection mechanism in different environments (indoor, outdoor). The mode selection mechanism is proposed using multi-criteria for decision-making technique, the mode selection mechanism based on Simple Additive Weighting (SAW) algorithm is used to wisely connect and switch between the available modes. The evaluation of the proposed mechanism for indoor environment and outdoor environment shows better performance based on user preferences.
\end{abstract}

Keywords-Device-to-Device, Mode Selection, Fifth Generation

\section{$1 \quad$ Introduction}

As wireless communication technology continues to evolve from one generation to another, the connection systems' requirements develop alongside to meet the expectations of users. Such expectations are brought about by the entire evolution process, which represents the ever-increasing demand for a higher system throughput. Nowadays, as the demand for higher transmission rates continues unabatedly, a new generation of wireless technologies is being introduced into the communication society to meet the insistent demands of users [1]. The key differences among the various generations of wireless communication systems (i.e., starting from the first generation $1 \mathrm{G}$ to the upcoming fifth generation systems) are manifested in variations in data rates, data security, latency, and the quality of services (QoS) [2][3]. These are often improved in successive generations for a more efficient system throughput, as well as QoS performance. 
In this paper, we focus on evaluating the proposed Multi-Criteria Mode-Selection for D2D (MCMS-D2D) mechanism. The main contributions of this paper can be summarized as follows:

- We evaluate the proposed MCMS-D2D mechanism MADM-based for assist the user and network to choose the best communication mode.

- We test the performance in the different environments; the first being an indoor environment and the second; an outdoor environment, showing better performances relative to the other approach mentioned before.

The rest of this paper is organized as follows:

\section{D2D Mode Selection Mechanism}

The random movement of mobile devices along with availability of a variety of communication modes at particular areas such as WLAN, LTE-A and D2D direct connection cause another challenge to establishing robust D2D connections. One of the relevant difficulties is how to keep providing a very stable connection for users even when the connection is switching between different modes either through D2D, LTE-A or WLAN [11]. This makes it difficult to select the best mode and requires wise switching between modes as well [8], [9]. For instance, when several networks are existing at a particular area like WLAN, LTE-A and D2D networks for instance, the mode selection procedure should be able to select the optimal connection mode to prevent the loss of connection.

As shown in Figure 1 the MCMS-D2D mechanism goes through a number of main steps. First, the MCMS-D2D mechanism defines the selected parameters to use it in the evaluation stage which are RSS, delay and bandwidth. The previous criteria are considered to help in selecting the suitable mode by giving every parameter the weight based on its importance based on users' preferences. Then the mechanism is finding the available modes to establish the connection. After finding the available modes the mechanism will do the calculation of the available modes based on the given weights in the previous step by adopting a simple additive weighting method (SAW) algorithm for accurate mode selection. Then the mechanism evaluates the modes to rank them based on the values. If the selected mode is D2D connection, directly it connects using direct D2D communication. If it is not D2D connection will be connected either to LTE-A or WLAN. 


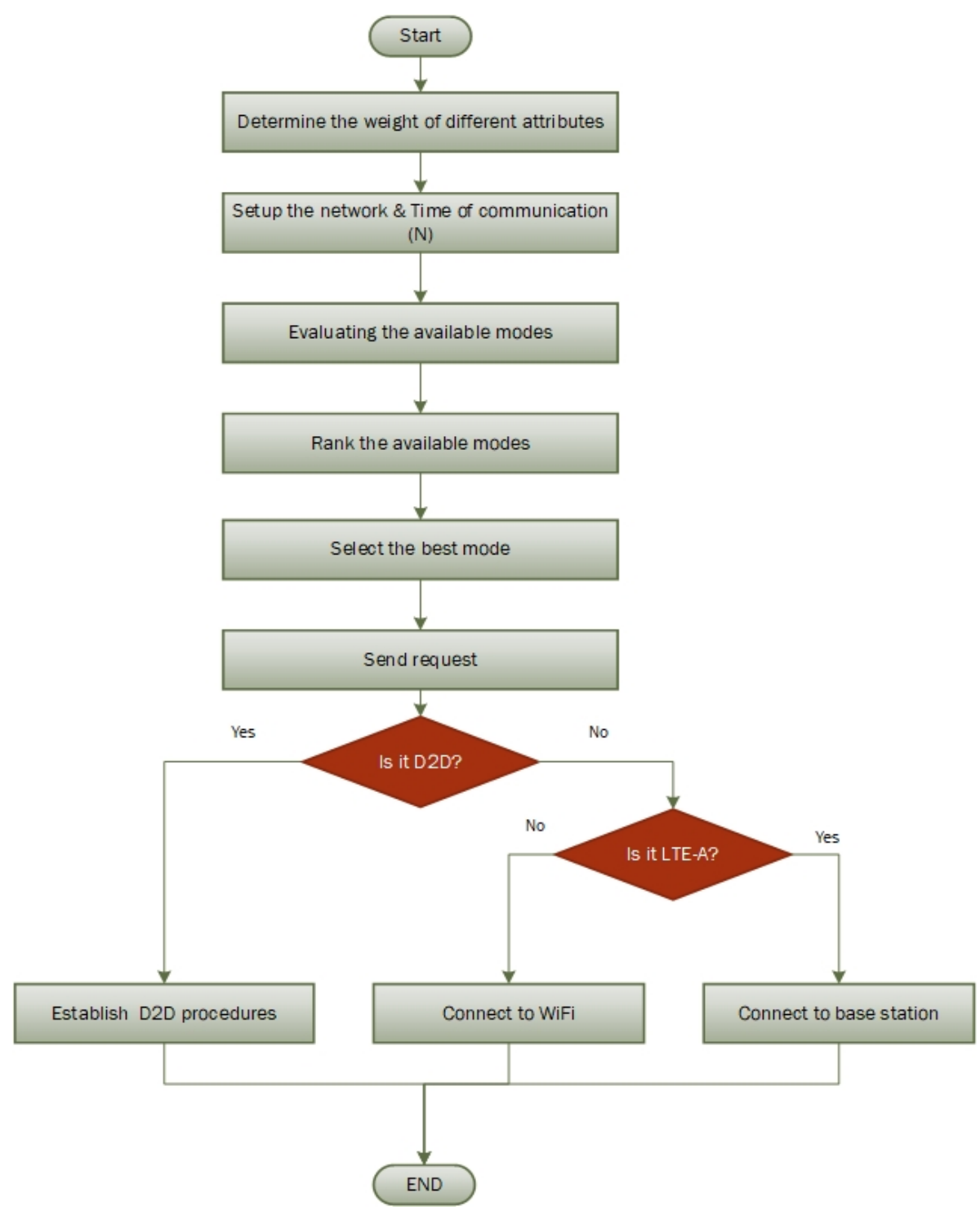

Fig. 1. MCMS-D2D Mechanism steps

\section{Simulation Result and Analysis}

In this paper, the both environment (i.e. indoor, outdoor) will be tested based on its condition. In general, the network contains many cells which have different mode of communication and different number of users. The proposed MCMS-D2D mechanism will assist in selecting the best available mode. It can switch between different technologies where the efficiency of selection can be different in diverse types of net- 
works' topologies as proposed in this paper outdoor environment and indoor environment which will have different conditions of the communications link. In future network, it is very important to give the user a stable connection every second and the ability to communicate in different modes to choose always best available mode of communication.

The indoor environment can exist in many cases such as shopping centers, offices, internal buildings or any airports halls. The indoor environment has open cubicle areas and walled areas, where the walls are different and the materials of walls are comprised of a mix of different types of materials, which may change the channel condition in this area [10]. For validation in this research, the study was applied on a shopping mall, which has six floors for offices and stores with two basement floors, and underground and many upper ground parking lots. Figure 2 shows the floor plan for the shopping centre.

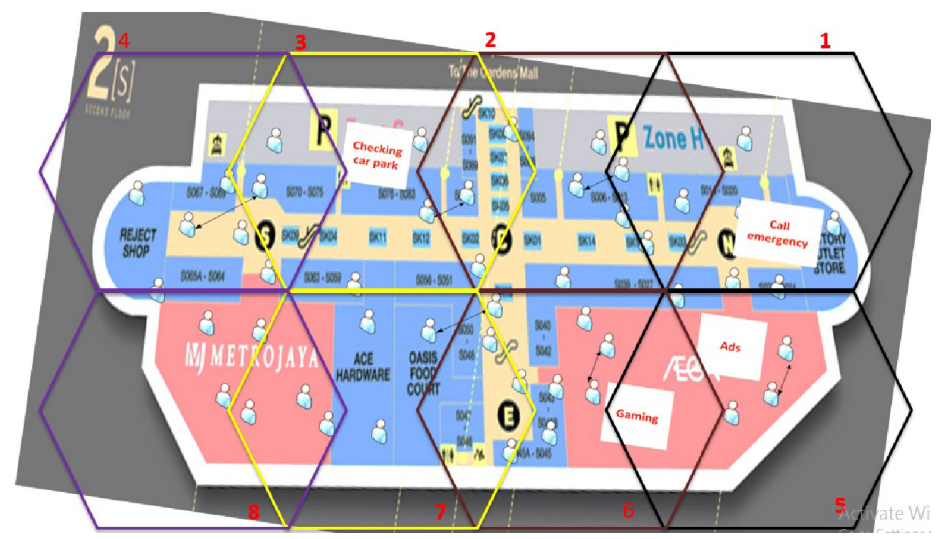

Fig. 2. Indoor environment

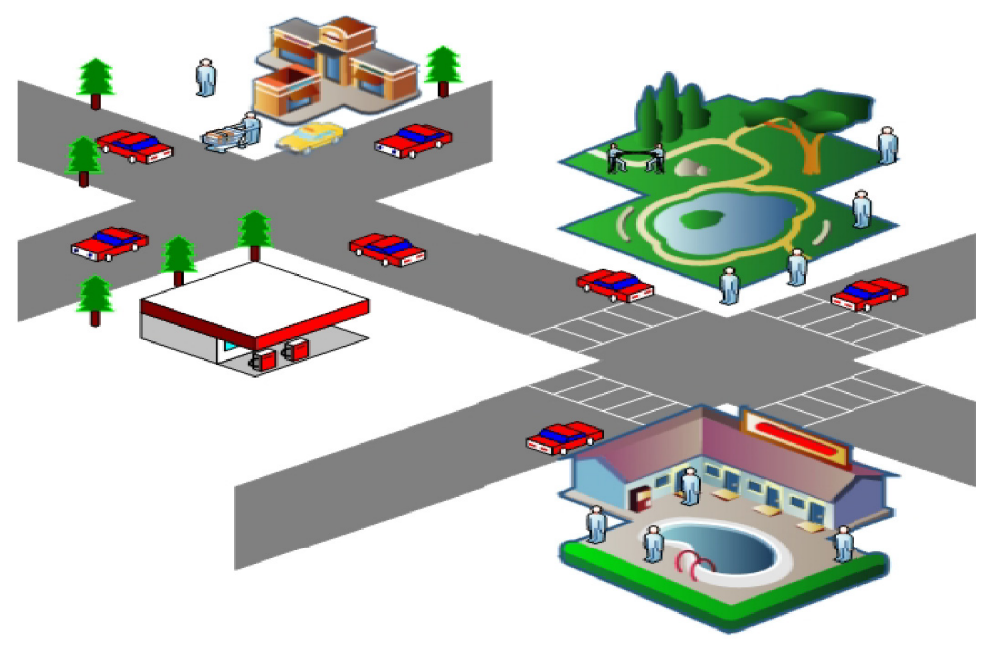

Fig. 3. Outdoor environment 
The outdoor environment is considered for this study such as a university campus as an outdoor scenario (open areas and outside the buildings' areas), or open sport areas, public parks and an outdoor shopping area. The outdoor areas may have different types of materials, which can affect the channel conditions such as trees, cars, buildings in the area, and weather conditions [12]. For the validation of this research, the study was applied in an open area around a shopping centre. Different activities take place in this area such as parking, sport activities, open restaurants, and cafes. Figure 3 shows the open area plan. A variety of activities can be performed in this area, which means that the users' conditions and status are different as they require a high data rate and a stable connection. For public safety purposes, it is therefore, highly important that users have good and stable connection, which can be supported by using the MCMS-D2D mechanism.

For the simulation, we consider aforementioned two types of environment and the user moving in network among different cells which consisting of 7 LTE-A cells and 6 WLAN access points. The inter-site distance of LTE-A network is $500 \mathrm{~m}$. The bandwidth is $5 \mathrm{MHz}$. The random distribution of 1000 users in the network is based on Poisson Point Process (PPP), which reflects ultra-dense users' distribution in the network. The evaluation steps of the proposed mechanism are carried out by MATLAB simulator.

In this evaluation stage, a comparison was made between the results of the proposed mechanism and other approaches' results when the user is moving in different environments. We compared theMCMS-D2D mechanism with RSS conventional approach which is focuses on received power

For both environments the evaluation part will compare the results between both approaches based on SINR, delay, throughput and delivery ratio.

\subsection{First Case: Outdoor Environment}

The implementation of this case showed the performance validation for the mode selection mechanism in the outdoor environment. This test underwent a lot of tests and simulations to ensure reliability of the validation and the results' values.

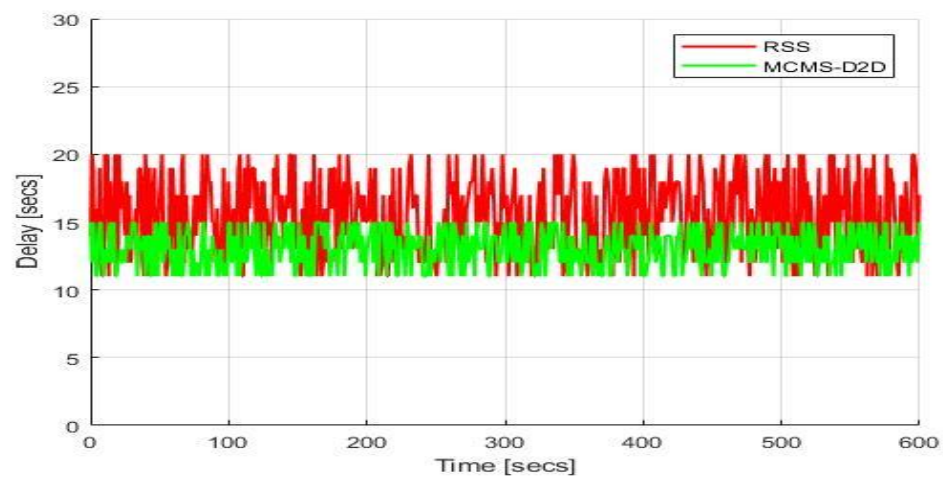

Fig. 4. Delay during Outdoor Phase 
The RSS approach is considered only on the highest received signal, whereby all devices may be connected to the same network, which can provide the highest received signal (this network can be LTE-A or D2D connection in this case). The connection ranged from 0 to 600 seconds. The RSS approach always showed higher delay than the MCMS-D2D mechanism. This happened because all packets were transmitted through one network, which can provide the highest RSS the packet queues. This network was highly congested (the buffer was full with the packet). Therefore, the buffering delay and scheduling delay were much higher than the MCMS-D2D mechanism. On the other hand, the MCMS-D2D mechanism distributed the load over the whole network for each data transmission, which evaluated the delay performance among available network modes as shown in Figure 4.

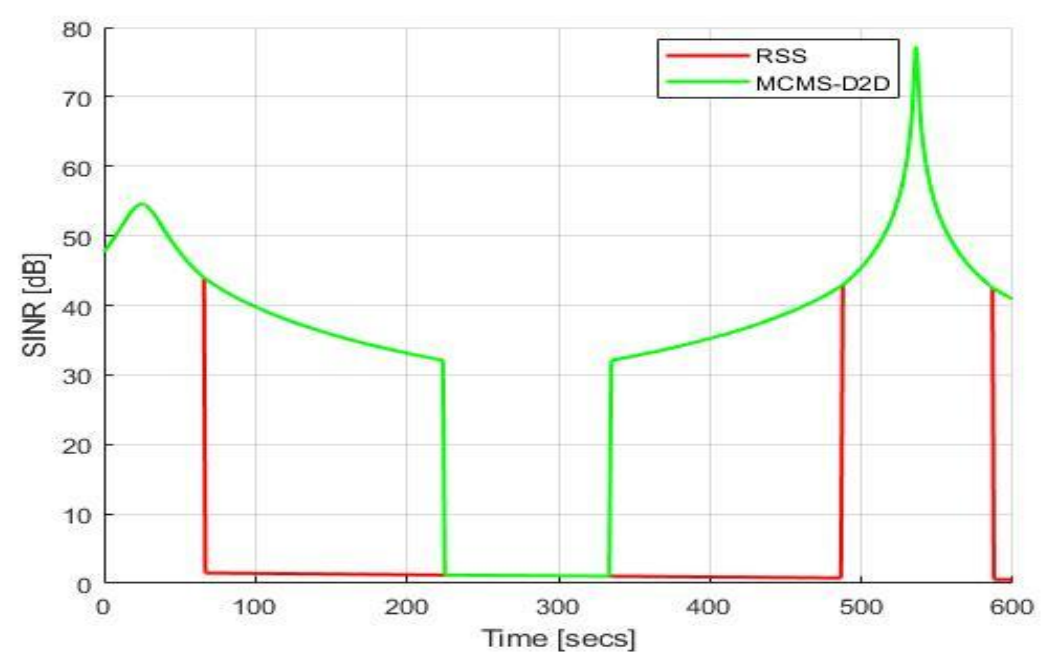

Fig. 5. SINR during Outdoor Phase

Based on Figure 5, the performance validation regarding SINR metric is clear, the time range from 0 to 70 seconds the SINR for both MCMS-D2D mechanism and RSS approach showed similar levels of performance and both of them were connected using D2D connection. From 70 to 220 seconds, the RSS value dropped near 0 . However, it was still connecting, while the MCMS-D2D mechanism kept connecting using D2D link with a better value in SINR compared to the RSS approach. From 220 to 330 seconds, both approaches showed a similar performance. From 330 to 480 seconds, MCMS-D2D mechanism showed more robust against interference affected by the connection using the D2D communication system. Accordingly, the performance in this period showed better performance in MCMS-D2D mechanism approach, which was roughly $45 \%$ better compared to RSS approach. It proved that the MCMSD2D mechanism selected the mode, which provided the lowest interference level. From 480 to 580 seconds, the connection used D2D communication system and both approaches showed a similar performance. From 580 to 600 seconds, the value of 
RSS approach dropped, while MCMS-D2D mechanism kept showing a better value than RSS. The results in this period showed better performance in MCMSD2D mechanism approach, which was roughly $40 \%$ better compared to the RSS approach. This clearly showed that the MCMS-D2D mechanism provided better performance than the RSS approach.

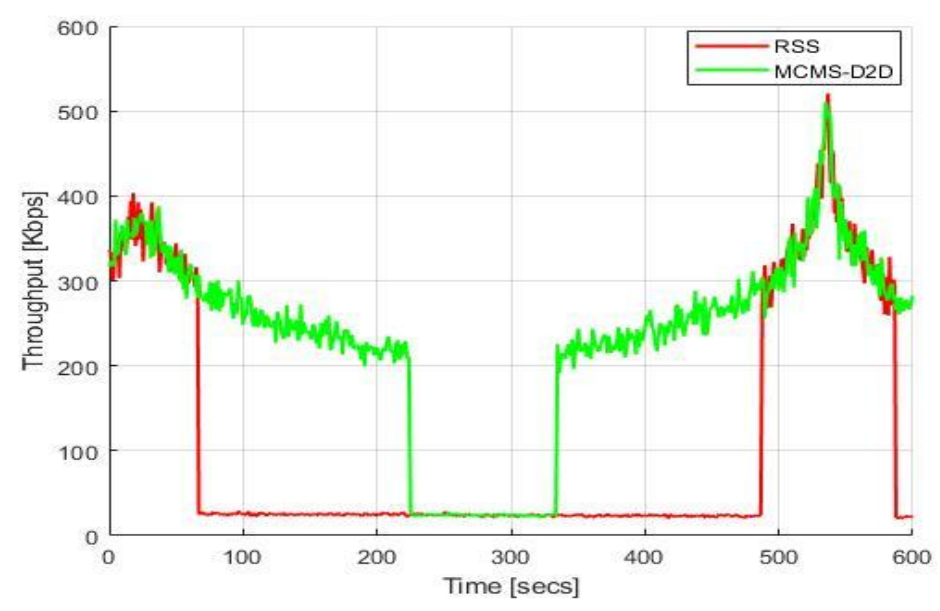

Fig. 6. Throughput Validation for Outdoor Phase

Based on Figure 6, the results can be divided into four phases as follows:

- Phase 1: From 0 to 70 seconds, both approaches showed similar performance in terms of connecting using the same mode, which they obtained it, because MCMSD2D mechanism and RSS approach had selected the D2D communication mode, while the mobile station started moving in the area and communicated with devices in the surrounding area.

- Phase 2: From 70 to 220 seconds, the mobile station was moving in a particular area, where the number of devices was not stable. The MCMS-D2D mechanism showed better performance than RSS as it kept connecting using D2D connection, whereas the RSS approach moved to connect using LTE for this period of time.

- Phase 3: From 220 to 330 seconds, both approaches showed similar performance in terms of connection. This is because both the MCMSD2D mechanism and RSS approach had selected the LTE communication mode.

- Phase 4: From 330 to 480 seconds, the MCMS-D2D mechanism selected to communicate using D2D link, while RSS kept connecting using LTE. The MCMSD2D mechanism showed better performance than RSS by connecting using D2D connection, whereas the RSS approach connected using LTE for this period of time.

- Phase 5: From 480 to 580 seconds, the mobile station kept moving in the network, where the number of devices increased. Both approaches showed similar perfor- 
mance in terms of connecting using the same mode, which is the D2D communication mode.

- Phase 6: From 580 to 600 seconds, the mobile station kept moving in the network, where the number of devices was different. The MCMS-D2D mechanism showed better performance than the RSS approach, because MCMS-D2D mechanism chose to keep connecting using the D2D connection, whereas the RSS approach chose to connect to different modes using LTE-A.

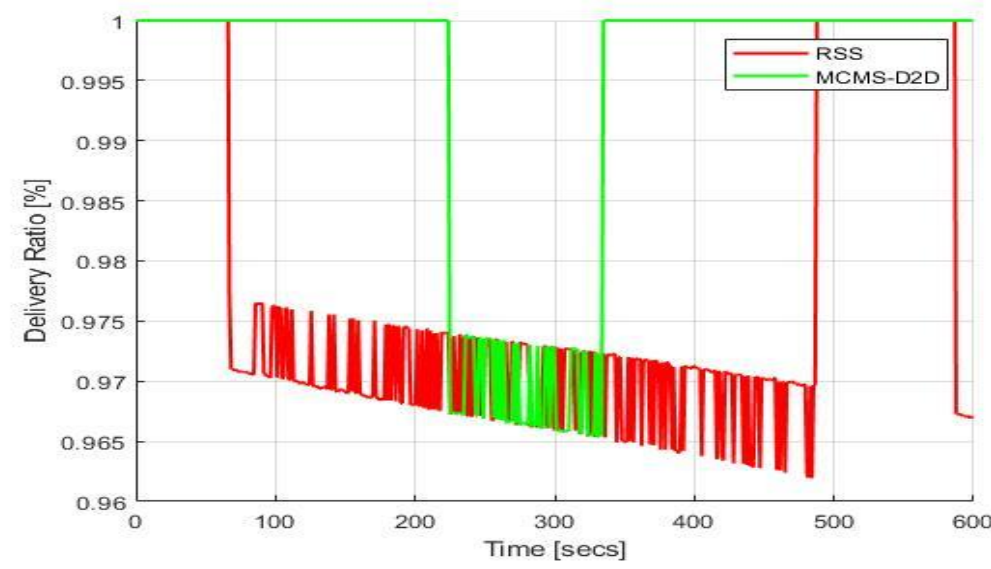

Fig. 7. Delivery Ratio for Outdoor Phase

The delivery ratio by using MCMS-D2D was found to be better than RSS approach most of the studied period or at least same to the RSS approach; it was between $96.5 \%$ and $100 \%$ as shown in Figure 7.

\subsection{Second Case: Indoor Environment}

The implementation of this case showed the performance validation for the mode selection mechanism in the outdoor environment. This test underwent a lot of tests and simulations to ensure reliability of the validation and the results' values. The mobile station kept moving in the network, where the number of devices was increased. The MCMS-D2D mechanism showed better performance than the RSS approach by $10 \%$. This is because MCMS-D2D mechanism chose to connect using D2D connection, whereas the RSS approach kept connecting to LTE for more time. 


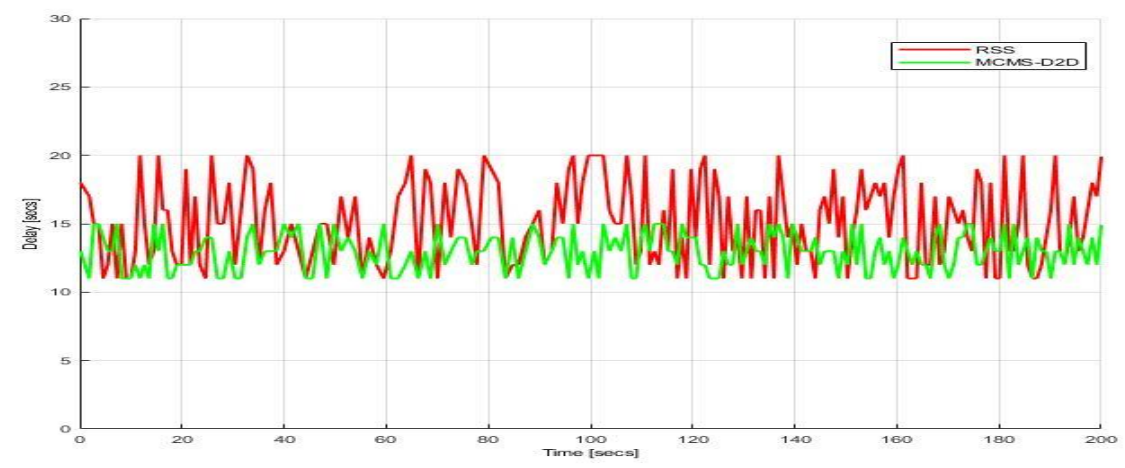

Fig. 8. Delay during Indoor Phase

Figure 8 shows the delay validation value for the proposed mechanism compared with that of the RSS approach. The RSS approach is considered only on the highest received signal, whereby all devices may be connected to the same network. Figure 5.9 shows that the connection ranged from 0 to 200 seconds, whereby the RSS approach always showed higher delay than MCMS-D2D mechanism. This happened because all packets were transmitted through one destination, which provided the highest RSS for the packet queues. This network was highly congested and, therefore, the buffering delay and the scheduling delay were much higher than that of the MCMS-D2D mechanism. On the other hand, the MCMS-D2D mechanism distributed the load over the whole network for each data transmission. This evaluated the delay performance among the available networks as shown in Figure 7.

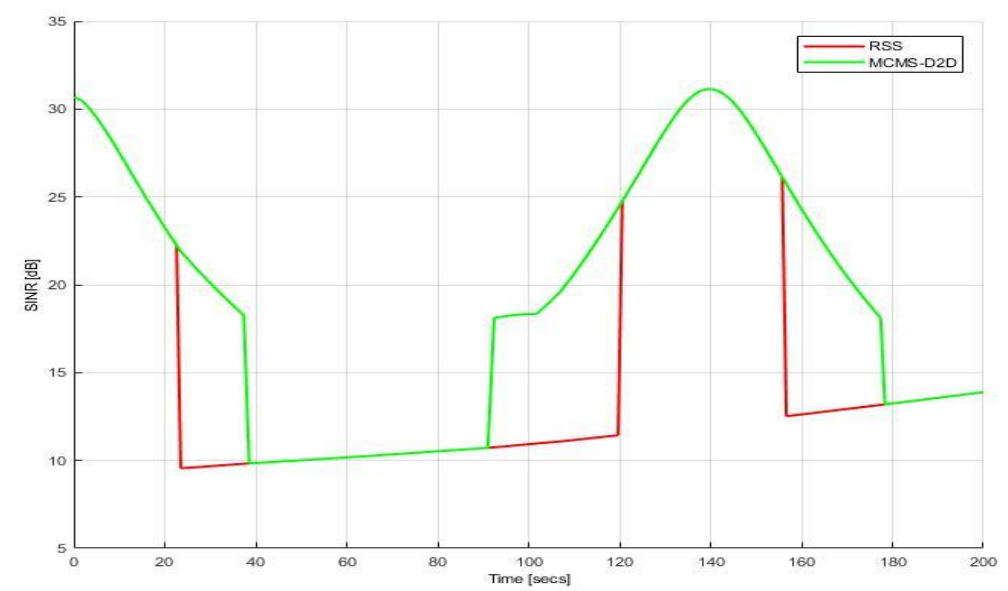

Fig. 9. SINR during Indoor Phase 
Figure 9 shows the performance validation regarding the SINR metric. From 0 to 25 seconds, the SINR for both RSS and MCMS-D2D mechanism showed similar performances and both of them chose to connect using D2D link, which gave a high value of the SINR. From 25 to 40 seconds, MCMS-D2D mechanism proved more robust against interference affected by keeping the connection using the D2D communication system, while the RSS dropped the value for this period. Accordingly, the performance during this period showed better performance in the MCMS-D2D mechanism, which was roughly $20 \%$ compared to the RSS approach. This proved that the MCMS-D2D mechanism considered selecting the mode, which provided the lowest interference level. From 40 to 90 seconds, the SINR dropped as the mobile station connected back to LTE network. However, it remained within the accepted level and showed similar performance value with the RSS approach for this period. From 90 to 120 seconds, the MCMS-D2D mechanism proved more robust against interference affected by keeping the connection using the D2D communication system, while the RSS dropped the value for this period. Therefore, the performance in this period showed better performance in the MCMS-D2D mechanism, which was roughly $25 \%$ compared to the RSS approach. This proved that the MCMS-D2D mechanism considered selecting the mode, which provided the lowest interference level. From 120 to 160 seconds, the SINR for both RSS and MCMS-D2D mechanism showed similar performance and both of them chose to connect using D2D link, which gave a high value of the SINR. From 160 to 180 seconds, the MCMS-D2D mechanism showed more robust against interference affected by keeping the connection using the D2D communication system, while the RSS drooped the value for this period. Accordingly, this period showed better performance for the MCMS-D2D mechanism, which was roughly $30 \%$ higher than that of the RSS approach. This proved that the MCMS-D2D mechanism considered selecting the mode, which provided the lowest interference level. Finally, from 180 to 200 seconds, the SINR dropped since the mobile station connected back to LTE network. However, it remained within the acceptable level and showed similar performance value with the RSS approach for this period.

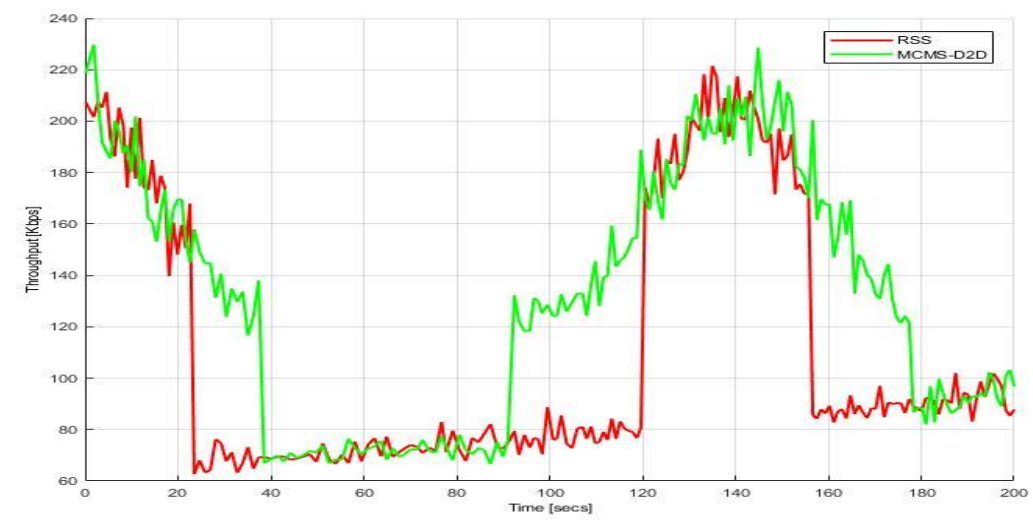

Fig. 10.Throughput during Indoor Phase 
Figure 10 shows the performance validation with regard to the throughput validation. From 0 to 25 seconds, the throughput for both RSS and MCMS-D2D mechanism showed similar performance and both of them chose to connect using the D2D link, which gave a high value of throughput. From 25 to 40 seconds, the MCMS-D2D mechanism proved more robust against interference affected by keeping the connection using the D2D communication system, while the RSS dropped the value for this period. Therefore, the performance in this period showed better performance in MCMS-D2D mechanism, which was roughly $20 \%$ better compared to the RSS approach. From 40 to 90 seconds, the throughput dropped as the mobile station connected back to LTE-A network. However, it remained within the accepted level and showed similar performance value with the RSS approach for this period. From 90 to 120 seconds, the MCMS-D2D mechanism proved to be more robust against interference affected by keeping the connection using the D2D communication system, while the RSS dropped the value for this period. Accordingly, the performance in this period showed better performance for the MCMS-D2D mechanism approach roughly $25 \%$ better compared to that of the RSS approach. This proved that the MCMS-D2D mechanism considered selecting the mode, which provided the highest throughput level. From 120 to 160 seconds, the throughput for both RSS and MCMS-D2D mechanism showed similar performance and both of them chose to connect using the D2D link, which gave a high value for throughput. From 160 to 180 seconds, the MCMSD2D mechanism showed to be more robust against interference affected by keeping the connection using the D2D communication system, while the RSS dropped the value for this period. Therefore, the performance in this period showed better performance in MCMS-D2D mechanism, which was roughly $30 \%$ better compared to the RSS approach. This proved that the MCMS-D2D mechanism considered selecting the best available mode, which provided the highest throughput level. Finally, from 180 to 200 seconds, the throughput dropped as the mobile station connected back to LTEA network. However, it remained within the accepted level and showed similar performance value with the RSS approach for this period.

The delivery ratio by using MCMS-D2D was found to be better or same to the RSS approach; it was between $99.5 \%$ and $100 \%$ as shown in Figure 11.

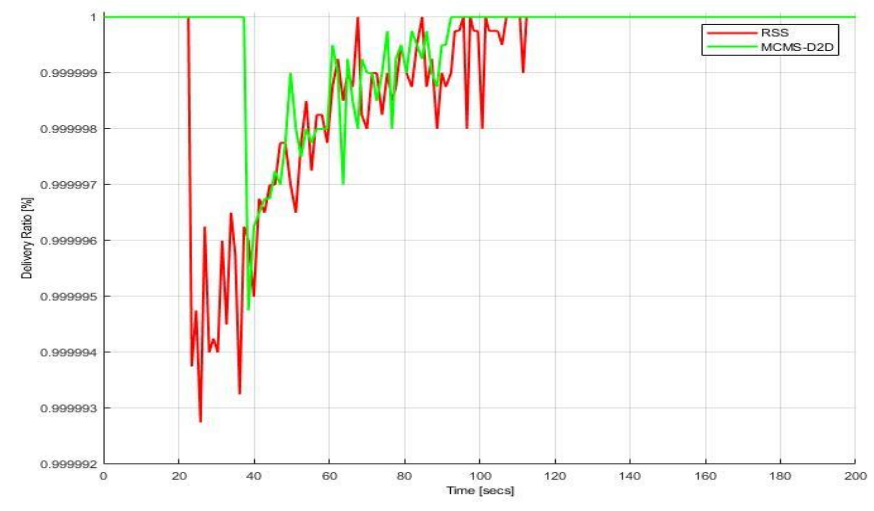

Fig. 11.Delivery Ratio Indoor Phase 


\section{Conclusion}

This study aimed to design and evaluate the MCMS-D2D mechanism that serve the D2D communication system. The first part discusses issues and challenges, which are related to have efficient mode selection mechanism to have efficient D2D communication system. An evaluation of the proposed mechanism is carried out in this paper in two different environments to guarantee that the MCMS-D2D mechanism is providing smooth and wise switching between different modes and choose always-best connection mode. The obtained results showed that the proposed mechanism outperform better than other approaches in terms of signal to noise ratio, delay delivery ratio and throughput. The proposed mechanism will assist users to meet their demands for different types of applications by serving them in any predictable situation and anywhere. Finally, the author has proposed approaches will serve users in the different environments, which can be find in different places such as shopping centre for indoor environment and Public Park for outdoor environment.

\section{Acknowledgement}

This work was supported by the Ministry of Higher Education, Malaysia, through FRGS Research Grant 13257.

\section{References}

[1] F. Zen Alden, S. Hassan, and A. Habbal, "Improving Network Performance by Enabling Device-to-Device Communication over Heterogeneous Networks."

[2] Ding, Z., Liu, Y., Choi, J., Sun, Q., Elkashlan, M., \& Poor, H. V. (2015). Application of non-orthogonal multiple access in LTE and 5G networks. arXiv preprint arXiv:1511.08610.

[3] A. Habbal, S. I. Goudar, and S. Hassan, "Context-Aware Radio Access Technology Selection in 5G Ultra Dense Networks," IEEE Access, vol. 5, no. Mmc, pp. 6636-6648, 2017.

[4] P. Sharma, "Evolution of mobile wireless communication networks-1G to $5 \mathrm{G}$ as well as future prospective of next generation communication network," Int. J. Comput. Sci. Mob. Comput., vol. 2, no. 8, pp. 47-53, 2013.

[5] J. G. Andrews et al., "What will 5G be?," IEEE J. Sel. AREAS Commun., vol. 32, no. 6, pp. 1065-1082, 2014 https://doi.org/10.1109/JSAC.2014.2328098

[6] A. Zakrzewska, S. Ruepp, and M. S. Berger, "Towards converged 5G mobile networkschallenges and current trends," in Proceedings of the 2014 ITU Kaleidoscope Academic Conference: Living in a converged world-Impossible without standards?, 2014, pp. 39-45. https://doi.org/10.1109/Kaleidoscope.2014.6858478

[7] F. Zenalden, S. Hassan, and A. Habbal, "Vertical handover in wireless heterogeneous networks," J. Telecommun. Electron. Comput. Eng., vol. 9, no. 1-2, 2017.

[8] S. Goudar, S. Hassan, A. Habbal. E. and Computer2017, "5G: The next wave of digital society challenges and current trends," journal.utem.edu.my. 
[9] Y. Huang, A. Nasir, A. A., Durrani, S., \& Zhou, X.2016, "Mode Selection, Resource Allocation, and Power Control for D2D-Enabled Two-Tier Cellular Network.," ieeexplore.ieee.org.

[10] Z. Yang, C. Wu, Z. Zhou, X. Zhang, X., Wang, X., \& Liu, Y. 2015, “Mobility increases localizability: A survey on wireless indoor localization using inertial sensors," dl.acm.org.

[11] S.V. Manikanthan, T.Padmapriya, "United Approach in Authorized and Unauthorized Groups in LTE-A Pro", Jour of Adv Research in Dynamical \& Control Systems, Vol. 10, 10-Special Issue, 2018, pp. (1137-1145).

[12] S. Rangan, T. Rappaport, Erkip, E. of the IEEE2014, "Millimeter-wave cellular wireless networks: Potentials and challenges," ieeexplore.ieee.org.

\section{$7 \quad$ Authors}

Feras Zen Alden is a Ph.D candidate specializing in wireless network in Universiti Utara Malaysia. His current area of research focuses on future internet and $5 \mathrm{G}$ mobile networks and its components such as D2D communication system. He received his master degree in ICT from Universiti Utara Malaysia in 2012. He received his bachelor degree in Telecommunication engineering in 2008 from IPU university in Syria.

Prof. Suhaidi Hassan is a tenure track professor of computing network and the founding chair of the InterNetWorks Research Laboratory, School of Computing, Universiti Utara Malaysia (UUM). He received his BS degree in computer science from State University of New York at Binghamton, New York, the MS degree in information science (with interest in telecommunication networks) from University of Pittsburgh, Pennsylvania, and his Ph.D. in computing (focussing on network performance engineering) from Leeds University, United Kingdom. He is the current President of the Internet Society Malaysia and the Internet Society Fellow alumnus to the Internet Engineering Task Force (IETF). He has authored and co-authored over 250 peer-reviewed technical publications and successfully supervised $25 \mathrm{Ph} . \mathrm{D}$. scholars in his research area of computing networks. In 2006, he led a task force for establishing the International Telecommunication Union (ITU)-UUM Asia-Pacific Centre of Excellence for Rural ICT Development which serves as a focal point for ITU's rural ICT development initiatives across the Asia-Pacific region. Prof. Hassan engages in various international forums, such as ICANN meetings, Internet Governance Forums, the IETF and the IEEE meetings. Apart from serving as reviewer for technical journals and conferences, Prof, Hassan serves as examiner for more than 100 doctoral and postgraduate theses in his research areas. He was also an IPv6 auditor for the Malaysian Communication and Multimedia Commission, the Malaysian ICT regulator.

Adib Habbal (SM'15) is a Professor (Associate) of Computer Engineering at Karabuk University, Turkey. Before joining Karabuk University in 2019, he was a senior lecturer at Universiti Utara Malaysia (ten years) and head of InterNetWorks Research Platform (three years). He also served as IEEE UUM Student Branch Founding Counselor and Executive Council Member of the Internet Society Malaysia Chapter. Dr. Habbal received his Ph.D. degree in Computer Science (specializing in Networked Computing) from Universiti Utara Malaysia. 
Dr. Habbal has received a number of recognitions from Universiti Utara Malaysia (UUM) for his outstanding educational and research activities including the Excellent Service Award (2010), Best Research Award (2014), Prolific Writer Award (2016) and many others. He has been the recipient of Internet Society Fellowship to the Internet Engineering Task Force (IETF), an IEEE Malaysia Section Best Volunteer Award, and an Asia-Pacific Advanced Network (APAN) Fellowship. Dr. Habbal is a senior member of the Institute of Electrical and Electronic Engineers (IEEE).

Dr. Habbal's research projects have been funded by several organizations, including IEEE R10, IEEE Malaysia Section, Internet society, Malaysian Ministry of Higher Education, Universiti Utara Malaysia and others. He has over 80 publications in journals and conference proceedings in the areas of Future Internet, and performance evaluation. His professional experience includes being a speaker at a number of renowned research conferences and technical meetings such as IEEE, internet2, APAN, and APRICOT, an editor for top tier and refereed journals, a technical program committee for IEEE conferences on computing networks as well as an examiner for postgraduate scholars in his research areas. His research interests include Future Internet protocols and architecture, 5Th Generation Mobile Networks, as well as Blockchain Technology and Digital Trust.

Article submitted 2019-01-18. Resubmitted 2019-02-24. Final acceptance 2019-03-15. Final version published as submitted by the authors. 